\title{
Inhibition of coxsackievirus B3 by RNA interference (RNAi)
} J Kurreck ${ }^{1}$, S Schubert ${ }^{1}$, D Werk ${ }^{1}$, D Rothe ${ }^{1}$, HP Grunert ${ }^{2}$, I Sipo ${ }^{3}$, H Fechner ${ }^{3}$, W Poller ${ }^{3}$, VA Erdmann ${ }^{1}$ and H Zeichhardt ${ }^{* 2}$

\author{
Address: ${ }^{1}$ Free University Berlin, Institute for Biochemistry, Berlin, Germany, ${ }^{2}$ Charité CBF, Department Virology, Berlin, Germany and ${ }^{3} \mathrm{Charité}$ \\ CBF, Department Cardiology, Berlin, Germany \\ * Corresponding author
}

from 2006 International Meeting of The Institute of Human Virology

Baltimore, USA. 17-2I November, 2006

Published: 21 December 2006

Retrovirology 2006, 3(Suppl I):P77 doi:I0.1I86/1742-4690-3-SI-P77

(C) 2006 Kurreck et al; licensee BioMed Central Ltd.

Here, we demonstrate the potential of RNAi to inhibit a picornavirus, coxsackievirus B3 (CVB3), a major causes of myocardial diseases. Targeting the 3D RNA dependent RNA polymerase (RdRP) with virus specific small interfering RNAs (siRNAs) resulted in a reduction of the virus titre by up to $90 \%$. A detailed analysis of the mechanism of virus silencing with strand-specific siRNAs revealed that only the viral plus-strand can be efficiently targeted in an RNAi approach, whereas the minus-strand is resistant to siRNA-mediated silencing. CVB3 is known to have a high error-rate during replication and can thus be expected to escape RNAi-silencing upon prolonged treatment. We have therefore developed an siRNA double expression vector (SiDEx), which generates two independent siRNAs simultaneously. This vector maintained its silencing capacity against the target RNA with an artificially introduced mutation in a reporter assay, whereas single siRNAexpression vectors lost their capacity to silence their respective target after substitution of a base in the centre of the target site. We will present a recently developed method to drastically facilitate and accelerate the generation of SiDEx vectors. Delivery of the siRNA double expression cassette into neonatal rat cardiomyocytes by an adenovirus-associated virus vector resulted in up to 370 fold reduction of the CVB3 titre. As an alternative approach to cope with the problem of viral escape, we silenced the coxsackievirus-adenovirus receptor on the host cell and achieved a significant reduction of CVB3 propagation. 